\title{
CALIDAD TECNOLÓGICA DE DOCE MÚSCULOS DE LLAMAS JÓVENES (Lama glama) CRIADAS BAJO UN SISTEMA DE CRIANZAEXTENSIVA
}

\author{
Technological Quality of Twelve Muscles from Young Llamas (Lama \\ glama) Reared under an Extensive Production System
}

\author{
Lindon W. Mamani-Linares ${ }^{1,3}$, Faustina Cayo $^{2}$, Carmen Gallo ${ }^{1}$
}

\section{Resumen}

Se compararon las características tecnológicas de doce músculos de llamas que fueron criadas al pastoreo. Las llamas $(n=10)$ fueron faenadas bajo procedimientos estándares de Bolivia. Las canales fueron refrigeradas durante 24 horas a $4{ }^{\circ} \mathrm{C}$. Los músculos fueron removidos de cada canal después del desposte. Cinco bifes de $2.54 \mathrm{~cm}$ de espesor fueron cortados de cada músculo. En cada músculo se determinó el $\mathrm{pH}_{24}$, color $\left(\mathrm{L}^{*}, \mathrm{a}^{*}, \mathrm{~b}^{*}\right)$, capacidad de retención de agua y fuerza de corte Warner-Bratzler (WBSF). No se observaron diferencias estadísticas en el $\mathrm{pH}$ entre los músculos. Los valores de L* (luminosidad) y b* (tenor de amarrillo) de los músculos L. thoracis, $L$. lumborum y Semimembranosus fueron mayores que en Triceps brachii y Psoas major $(\mathrm{p}<0.05)$. El porcentaje de pérdida por cocción fue menor en los músculos Infraspinatus y Longissimus thoracis ( $\mathrm{p}<0.05)$. El valor WBSF de los músculos Semimembranosus, Semitendinosus, Quadriceps femoris y Biceps femoris fue mayor $(\mathrm{p}<0.05)$ y del músculo Infraspinatus fue el más bajo $(\mathrm{p}<0.05)$. La textura (WBSF), color y capacidad de retención de agua variaron entre los músculos.

Palabras clave: calidad tecnológica, músculos, Lama glama

\section{Abstract}

This study compared the technological quality of twelve muscles of llama that were raised in a grazing system. The llamas $(n=10)$ were slaughtered using Bolivian standard procedures. Carcasses were stored for $24 \mathrm{~h}$ in a cold room $\left(4^{\circ} \mathrm{C}\right)$. Muscles were removed from the left side of each carcass in the slaughter house. Five steaks $(2.54 \mathrm{~cm}$ thick $)$ were cut from each muscle. In each muscle was determined $\mathrm{pH}$, colour $\left(\mathrm{L}^{*}, \mathrm{a}^{*}, \mathrm{~b}^{*}\right)$, water

\footnotetext{
${ }^{1}$ Instituto de Ciencia Animal, Facultad Ciencias Veterinarias, Universidad Austral de Chile, Valdivia, Chile

${ }^{2}$ Carrera de Medicina Veterinaria y Zootecnia, Universidad Pública de El Alto, La Paz, Bolivia

${ }^{3}$ E-mail: willymlmvzupea_2@hotmail.com
} 
holding capacity (WHC) and Warner-Bratzler shear-force (WBSF). No statistical difference was found in $\mathrm{pH}$ between muscles. Values of $\mathrm{L}^{*}$ (lightness) and $\mathrm{b}^{*}$ (tenor of yellow) in L. thoracis, L. lumborum and Semimembranosus were higher than those in Triceps brachii and Psoas major ( $\mathrm{p}<0.05$ ). The cooking loss percentage was lower in Infraspinatus and Longissimus thoracis $(\mathrm{p}<0.05)$. The WBSF value in the Semimembranosus, Semitendinosus, Quadriceps femoris and Biceps femoris muscles was higher $(\mathrm{p}<0.05)$ and in Infraspinatus was the lowest $(\mathrm{p}<0.05)$. Tenderness (WBSF), colour, and water holding capacity varied between muscles.

Key words: technological quality, muscles, Lama glama

\section{INTRODUCCIÓN}

En los últimos años, se ha puesto énfasis en la búsqueda de métodos de evaluación de canales que permitan no solo predecir rendimientos (rendimiento de cortes nobles, proporción de músculo y grasa, etc.), sino también las características organolépticas y tecnológicas de la carne, a fin de poder estimar la calidad final del producto a nivel de consumidor (Gallo, 2010).

Según Lawrie y Ledward (2006), los parámetros más importantes considerados en la evaluación de la calidad de la carne son apariencia, jugosidad, terneza y sabor. La carne debe tener un color atractivo que debe ser uniforme a lo largo de todo el corte. El color de la carne está relacionado con los niveles de mioglobina presentes en el músculo. La carne también debe tener un adecuado marmoleo (grasa intramuscular), la cual mejora la jugosidad, terneza y sabor de la carne. La capacidad de retención de agua es un factor que también determina la jugosidad de la carne y se define como la capacidad de la carne para retener su agua durante la aplicación de fuerzas externas, tales como corte, enfriamiento, calentamiento, trituración o prensado. Si se observa exceso de agua en la parte inferior del paquete de venta al por menor, puede dar lugar a un producto seco cuando es cocinado (Muchenje et al., 2009).

En el bovino, los músculos de la carcasa se diferencian en su composición química
(Jeremiah et al., 2003), color, tamaño, forma (Bass et al., 2009) y terneza (Belew et al., 2003). Estas características influyen en la manera en que se utiliza la carne. En el comercio minorista, los precios altos se ofrecen para los cortes caracterizados por una terneza deseable, tales como lomo o filete, pero otros del cuello y algunos de la pierna se venden a menudo como productos molidos a un precio más bajo que los filetes o el bife (Belew et al., 2003).

Debido a la variedad de sistemas de producción, faena y prácticas de procesamiento de carne en los países del orbe, así como las distintas prioridades de los consumidores, los resultados de las características tecnológicas de músculo de bovinos u otras especies en un país no necesariamente son directamente aplicables en otros países. Es por ello que el objetivo de este trabajo fue determinar las características tecnológicas de diferentes músculos de llamas jóvenes criadas bajo un sistema extensivo en el altiplano boliviano.

\section{Materiales y Métodos}

\section{Animales, Carcasas y Muestras}

Diez llamas machos enteros del genotipo Kh'ara, de 18 a 24 meses de edad, fueron usados en el estudio. Los animales fueron adquiridos de un predio del altiplano boliviano durante la temporada de lluvias, donde su alimentación era basada en pastura nativa. 
Las llamas fueron pesadas y transportadas a una planta faenadora comercial y mantenidas en ayunas, pero con disponibilidad de agua por 12 horas previas a la faena. El faenado se hizo de acuerdo al procedimiento estándar de Bolivia donde las carcasas fueron refrigeradas por $24 \mathrm{~h}$ en sala de frío $\left(2-4{ }^{\circ} \mathrm{C}\right)$. Se removieron los cortes del lado izquierdo de cada una de las carcasas siguiendo los estándares de UNECE (2008): chingolo (0124), centro de carnaza de paleta (0122), bife ancho sin tapa (0125), bife angosto (0181), lomo (0182), cuadril (0178), nalga de adentro (0173), peceto (0172), cuadrada (0177) y bola de lomo (0175).

Los siguientes músculos individuales fueron retirados de los cortes comerciales: Supraspinatus, Infraspinatus, Triceps brachii, Longissimus thoracis, Longissimus lumborum, Psoas major, Gluteus medius, Semitendinosus, Biceps femoris, Quadriceps femoris, Semimembranosus, Aducctor. Estos músculos fueron cortados manualmente en bifes de $2.54 \mathrm{~cm}$ de espesor. Un bife de cada músculo fue asignado a 2 días de periodo de maduración $\left(2-4^{\circ} \mathrm{C}\right)$ y posteriormente congelado a $-20{ }^{\circ} \mathrm{C}$ para determinar pérdida de cocción y a la prueba de Warner-Bratzler shear force (WBSF) dentro de los siguientes 30 días. Los restantes bifes ( $24 \mathrm{~h}$ post faena) de cada músculo fueron envasados y congelados a $-20{ }^{\circ} \mathrm{C}$ (para determinar composición química, y cuyos resultados no son parte de esta publicación).

\section{Calidad Tecnológica}

Los bifes se descongelaron a $4{ }^{\circ} \mathrm{C} \mathrm{du}-$ rante 24 horas previas a la medición de los parámetros bajo evaluación, estimándose la pérdida por descongelación (pérdida de descongelado $=$ [peso congelado - peso descongelado] x 100/peso congelado), expresándose en porcentaje.

Color del músculo. El color de los músculos fue medido a las $24 \mathrm{~h}$ post faena sobre la superficie transversal de los cortes de los músculos en bifes y expuestos al ambiente por un periodo de $1 \mathrm{~h}$ a $4{ }^{\circ} \mathrm{C}$. Se utilizó un espectrocolorímetro Miniscan XE Plus $\left(\right.$ Hunterlab $^{\circledR}$ ) con escala Lab y con una longitud de onda entre los 400 y $700 \mathrm{~nm}$ para determinar los valores de «L» (luminosidad), «a» (tenores de rojo-verde) $\mathrm{y}$ «b» (tenores de amarillo-azul).

$p H$. El pH de los músculos se determinó a las $24 \mathrm{~h}$ post faena por medio de un peachímetro con electrodo de penetración (Hanna ${ }^{\circledR}$, HI 98230) introducido al centro de los bifes utilizados para medir color.

Pérdida de cocción. Para la determinación de las pérdidas por cocción, las muestras de los músculos descongelados ( $1^{\text {er }}$ grupo de bifes) fueron sometidas a cocción en un horno eléctrico a convección forzada $\left(\right.$ Trotter $^{\circledR}$ ) a $170{ }^{\circ} \mathrm{C}$. La cocción finalizó cuando la temperatura interna de cada bife alcanzó los $70^{\circ} \mathrm{C}$ al centro de la carne, para lo cual se usaron termómetros Brannan ${ }^{\circledR}$. La pérdida por cocción se calculó como la diferencia entre el peso de la muestra antes y después de la cocción, expresada como porcentaje del peso de la muestra inicial (AMSA, 1995).

Textura (Warner-Bratzler shear force, WBSF). Se midió utilizando el equipo WarnerBratzler (Salter ${ }^{\circledR}$ ), determinando la fuerza de cizalla, que corresponde a la fuerza $(\mathrm{kg})$ requerida para cortar un cilindro de carne cocida. Se utilizó el bife en que se determinó la pérdida por cocción. Las mediciones de fuerza de cizalla se realizaron en cilindros de carne extraídos de cada bife con un sacabocados de $1.2 \mathrm{~cm}$ de diámetro. El corte de cizalla se realizó al centro de cada cilindro (Beltrán y Roncalés, 2000).

\section{Análisis Estadístico}

La calidad tecnológica de los músculos fue comparada a través del modelo lineal general (GLM) de Statistix para Windows v. 8.0, y en casos de diferencia estadística se empleó la prueba de Tukey HSD, considerando $\mathrm{p}<0.05$ como diferencia estadística. 


\section{Resultados y Discusión}

En el Cuadro 1 se observa que el valor promedio de $\mathrm{pH}$ fue similar entre los doce músculos y que, además, se encuentran dentro de los rangos normales $(<5.8)$. En alpacas, Salvá et al. (2009) reportaron un $\mathrm{pH}$ promedio para carne de alpaca de 5.63, mientras que Cristofanelli et al. (2004) reportan valores de pH de 5.60 y 5.57 a las $24 \mathrm{~h}$ del sacrificio en llamas y alpacas, respectivamente. En bovinos, el $\mathrm{pH}$ es un parámetro importante relacionado con la susceptibilidad de la carne a su deterioro y se usa para decidir sobre el tipo de procesamiento al que se va a destinar la carne (Wirth, 1987).

El pH depende de factores como el estrés ante-mortem, factores genéticos, condiciones post-mortem, sexo, clase o categoría de animal, tipo de alimentación y región anatómica en que se mide, entre otros (Tarrant y Grandin 1993; Herrera, 2008; Apaoblaza et al., 2008). Cuando el animal se somete a estrés prolongado y consume sus reservas de glucógeno, no hay glicólisis anaerobia post-mortem y la carne presenta la condición conocida como «corte oscuro» (Hood y Tarrant, 1980). En el estudio de Almonacid (2003), los cortes comerciales lomo vetado (Longissimus thoracis), lomo liso (Longissimus thoracis et lumborum), pollo ganso (Semitendinosus), abastero (Gastrocnemius), asado del carnicero (Subscapularis), posta negra (Semimembranosus) y tapabarriga (Obliquus internus abdominis) presentaron promedios de $\mathrm{pH}$ mayores o iguales a 5.8 en las canales con corte oscuro.

Los camélidos parece ser poco susceptibles a la pérdida de calidad de la carne debida al estrés y no suele presentar valores de $\mathrm{pH}$ elevados. En comparación a otros rumiantes, llamas y alpacas mantienen altas concentraciones de glucosa en sangre, que pueden ser explicadas por una lenta respuesta a insulina y moderada resistencia a insulina, algo similar a una condición de diabetes $(\mathrm{Ce}-$ bra et al., 2001a,b).

Los músculos presentaron diferencias estadísticas en color (Cuadro 1). Los valores de $\mathrm{L}^{*}$ y b* de los músculos $L$. thoracis, $L$. lumborum y Semimembranosus fueron mayores $(\mathrm{p}<0.05)$ a aquellos de Triceps brachii y Psoas major. Los resultados hallados en el color de Longissimus thoracis y Longissimus lumborum del presente estudio fueron similares en $\mathrm{L}^{*}$, pero diferentes en $\mathrm{a}^{*}$ y en $\mathrm{b}^{*}$ a los reportados por Salvá et al. (2009) en carne de alpaca, lo que puede ser debido al mayor contenido de grasa intramuscular (2.1\%) y al suplemento de grano recibido por las alpacas en semanas previas al sacrificio.

Los valores de $\mathrm{a}^{*} \mathrm{y} \mathrm{b}^{*}$ en las llamas están dentro de rangos reportados para bovinos criados a pastoreo (de 11.1 a 23.6 para $a^{*}$ y de 6.1 a 11.3 para $\left.b^{*}\right)$, pero presenta valores más bajos de luminosidad $\left(\mathrm{L}^{*}\right)$ en relación a reportes de otros autores que van entre 33.2 a 41.0 (Realini et al., 2004; Muchenje et al., 2009; Orellana et al., 2009). La intensidad de $b^{*}$ se ve influida por el contenido y el color de la grasa intramuscular presente en la carne. Asimismo, una mayor concentración de caroteno se encuentra en animales de mayor edad y cuando el forraje es la principal fuente de alimentación en sistemas de pastoreo (Priolo et al., 2002).

Los valores de pérdida en el descongelado fueron más bajos para Triceps brachii y más altos para Longissimus lumborum, Gluteus medius, Semimembranosus, Adductor y Quadriceps femoris (Cuadro 2, $\mathrm{p}<0.05)$. Pobre capacidad de retención de agua (WHC) resulta en mayor pérdida por goteo y escurrimiento. Altas pérdidas por goteo y escurrimiento pueden representar importantes pérdidas de peso en canal y cortes, afectando la producción y calidad del procesamiento de carne (Savage et al., 1990; Wright et al., 2005; Pearce et al., 2011). 
Cuadro 1. Valores promedios ( \pm desviación estándar) para $\mathrm{pH}$ y color de doce músculos de carcasas de llamas refrigeradas por $24 \mathrm{~h}$ en sala de frío $\left(4^{\circ} \mathrm{C}\right)$

\begin{tabular}{lcccc}
\hline Músculos & $\mathrm{pH}_{24}$ & $\mathrm{~L}^{*}$ & $\mathrm{a}^{*}$ & $\mathrm{~b}^{*}$ \\
\hline Supraspinatus & $5.44 \pm 0.03$ & $27.63 \pm 3.16^{\mathrm{b}}$ & $11.87 \pm 0.72^{\mathrm{a}}$ & $8.61 \pm 0.58^{\mathrm{ab}}$ \\
Infraspinatus & $5.56 \pm 0.09$ & $30.06 \pm 7.14^{\mathrm{a}}$ & $11.54 \pm 1.33^{\mathrm{ab}}$ & $9.02 \pm 0.95^{\mathrm{a}}$ \\
Triceps brachii & $5.35 \pm 0.06$ & $25.31 \pm 0.59^{\mathrm{c}}$ & $11.58 \pm 0.95^{\mathrm{a}}$ & $8.51 \pm 0.66^{\mathrm{ab}}$ \\
Longissimus thoracis & $5.40 \pm 0.03$ & $33.92 \pm 1.55^{\mathrm{a}}$ & $11.81 \pm 0.59^{\mathrm{a}}$ & $10.26 \pm 0.52^{\mathrm{a}}$ \\
Longissimus lumborum & $5.47 \pm 0.12$ & $33.31 \pm 2.52^{\mathrm{a}}$ & $10.12 \pm 0.56^{\mathrm{b}}$ & $9.34 \pm 0.52^{\mathrm{a}}$ \\
Psoas major & $5.38 \pm 0.05$ & $26.51 \pm 2.08^{\mathrm{c}}$ & $11.44 \pm 0.38^{\mathrm{ab}}$ & $7.66 \pm 0.36^{\mathrm{b}}$ \\
Gluteus medius & $5.30 \pm 0.07$ & $31.50 \pm 2.06^{\mathrm{a}}$ & $10.19 \pm 0.51^{\mathrm{b}}$ & $8.65 \pm 0.64^{\mathrm{ab}}$ \\
Semimembranosus & $5.28 \pm 0.05$ & $32.30 \pm 0.59^{\mathrm{a}}$ & $10.92 \pm 0.34^{\mathrm{ab}}$ & $9.52 \pm 0.45^{\mathrm{a}}$ \\
Semitendinosus & $5.42 \pm 0.05$ & $28.47 \pm 1.74^{\mathrm{b}}$ & $10.68 \pm 0.56^{\mathrm{ab}}$ & $7.63 \pm 0.62^{\mathrm{b}}$ \\
Adductor & $5.27 \pm 0.06$ & $27.79 \pm 1.52^{\mathrm{b}}$ & $11.11 \pm 0.71^{\mathrm{ab}}$ & $8.95 \pm 0.75^{\mathrm{ab}}$ \\
Quadriceps femoris & $5.36 \pm 0.04$ & $29.63 \pm 1.30^{\mathrm{ab}}$ & $11.52 \pm 0.26^{\mathrm{ab}}$ & $9.17 \pm 0.21^{\mathrm{a}}$ \\
Biceps femoris & $5.32 \pm 0.02$ & $28.88 \pm 1.08^{\mathrm{b}}$ & $11.65 \pm 0.34^{\mathrm{a}}$ & $9.20 \pm 0.22^{\mathrm{a}}$ \\
\hline
\end{tabular}

$a, b, c$ Superíndices diferentes dentro de columnas indican diferencias significativas $(p<0.05)$

"L" (luminosidad), "a" (tenores de rojo-verde) y "b" (tenores de amarillo-azul)

Las pérdidas por cocción fueron menores en los músculos Infraspinatus, Longissimus thoracis y mayores en los músculos Supraspinatus, Triceps brachii, Gluteus medius, Semimembranosus, Semitendinosus, Quadriceps femoris y Biceps femoris (Cuadro 2, $\mathrm{p}<0.05$ ). Sin embargo, los porcentajes de pérdida de cocción de todos los músculos fueron más bajos que los reportados para Longisimus thoracis y lumborum en la alpaca (Salvá et al., 2009) y en novillos (Duckett et al., 2007), pero similar a reportes en corderos (16.7\%) y chivos (17.0\%) (Lee et al., 2008).

Los valores WBSF de los músculos Semimembranosus, Semitendinosus, Quadriceps femoris y Biceps femoris fueron los más altos y del músculo Infraspinatus fue el más bajo ( $\mathrm{p}<0.05)$. Trabajos realizados con carne (Longissimus dorsi) de alpaca registran valores más altos $\left(4.67-6.06 \mathrm{~kg} / \mathrm{cm}^{2}\right)$ (Polidori et al., 2007; Salvá et al., 2009).

La WBSF es un indicador de la terne$\mathrm{za}$, que es definida como la facilidad con que la carne se deja masticar. Su fuente de variación puede atribuirse a la edad del animal, sexo, peso, raza y estrés ante-mortem (Muchenje et al., 2009). Valores altos están relacionados con alta actividad de calpastatina, baja grasa intramuscular, baja de fragmentación miofibrilar, altos niveles de colágeno totales y bajo colágeno soluble o la combinación de estos factores (Nakamura $e t$ al., 2010; Duarte et al., 2011). Belew et al. (2003) clasifican los músculos como "muy tiernos" (WBS <3.2 kg/cm²), "tiernos" (3.2 <WBS <3.9 kg/cm²), "intermedios" (3.9 $\mathrm{kg}<$ WBS $<4.6 \mathrm{~kg} / \mathrm{cm}^{2}$ ) y "duros" (WBS $>4.6$ $\mathrm{kg} / \mathrm{cm}^{2}$ ), y los doce músculos bajo estudio serían considerados como muy tiernos. 
Cuadro 2. Valores promedio ( \pm desviación estándar) para Capacidad de Retención de Agua y WBSF de doce músculos de carcasas de llamas criadas en sistemas de pastoreo extensivo

\begin{tabular}{lccccc}
\hline \multirow{2}{*}{ Músculos } & \multicolumn{5}{c}{ Pérdidas } \\
\cline { 2 - 5 } & $\begin{array}{c}\text { Por } \\
\text { descongelado } \\
(\%)\end{array}$ & $\begin{array}{c}\text { Por } \\
\text { evaporación } \\
(\%)\end{array}$ & $\begin{array}{c}\text { Por goteo } \\
(\%)\end{array}$ & $\begin{array}{c}\text { Por cocción } \\
(\%)\end{array}$ & $\begin{array}{c}\text { WBSF } \\
\left(\mathrm{kg} / \mathrm{cm}^{2}\right)\end{array}$ \\
\hline Supraspinatus & $4.3 \pm 0.8^{\mathrm{b}}$ & $16.4 \pm 1.8^{\mathrm{ab}}$ & $3.0 \pm 0.6^{\mathrm{a}}$ & $19.4 \pm 2.0^{\mathrm{a}}$ & $1.6 \pm 0.2^{\mathrm{c}}$ \\
Infraspinatus & $4.2 \pm 1.0^{\mathrm{b}}$ & $13.6 \pm 0.9^{\mathrm{b}}$ & $2.5 \pm 0.3^{\mathrm{ab}}$ & $16.0 \pm 0.9^{\mathrm{b}}$ & $1.3 \pm 0.3^{\mathrm{d}}$ \\
Triceps brachii & $2.6 \pm 0.9^{\mathrm{c}}$ & $17.8 \pm 1.6^{\mathrm{a}}$ & $2.5 \pm 0.7^{\mathrm{ab}}$ & $20.4 \pm 1.7^{\mathrm{a}}$ & $1.8 \pm 0.2^{\mathrm{bc}}$ \\
Longissimus thoracis & $6.3 \pm 1.3^{\mathrm{ab}}$ & $14.3 \pm 0.7^{\mathrm{b}}$ & $1.4 \pm 0.7^{\mathrm{b}}$ & $15.7 \pm 1.3^{\mathrm{b}}$ & $1.8 \pm 0.2^{\mathrm{bc}}$ \\
Longissimus lumborum & $8.2 \pm 1.2^{\mathrm{a}}$ & $15.9 \pm 2.1^{\mathrm{ab}}$ & $1.3 \pm 0.5^{\mathrm{b}}$ & $17.2 \pm 2.3^{\mathrm{b}}$ & $2.2 \pm 0.3^{\mathrm{ab}}$ \\
Psoas major & $4.8 \pm 1.5^{\mathrm{b}}$ & $14.8 \pm 2.2^{\mathrm{b}}$ & $1.6 \pm 0.5^{\mathrm{ab}}$ & $16.4 \pm 2.2^{\mathrm{b}}$ & $1.8 \pm 0.2^{\mathrm{bc}}$ \\
Gluteus medius & $7.6 \pm 0.4^{\mathrm{a}}$ & $16.9 \pm 1.9^{\mathrm{ab}}$ & $2.5 \pm 0.2^{\mathrm{ab}}$ & $19.4 \pm 1.8^{\mathrm{a}}$ & $2.0 \pm 0.2^{\mathrm{b}}$ \\
Semimembranosus & $9.0 \pm 2.0^{\mathrm{a}}$ & $17.3 \pm 1.6^{\mathrm{a}}$ & $2.7 \pm 0.7^{\mathrm{ab}}$ & $20.0 \pm 1.4^{\mathrm{a}}$ & $2.6 \pm 0.5^{\mathrm{a}}$ \\
Semitendinosus & $2.5 \pm 1.0^{\mathrm{c}}$ & $16.1 \pm 2.7^{\mathrm{ab}}$ & $2.7 \pm 0.8^{\mathrm{ab}}$ & $19.1 \pm 2.9^{\mathrm{a}}$ & $2.5 \pm 0.3^{\mathrm{a}}$ \\
Adductor & $8.3 \pm 1.6^{\mathrm{a}}$ & $15.6 \pm 1.0^{\mathrm{ab}}$ & $1.5 \pm 0.3^{\mathrm{ab}}$ & $17.1 \pm 1.1^{\mathrm{b}}$ & $2.1 \pm 0.2^{\mathrm{b}}$ \\
Quadriceps femoris & $7.7 \pm 0.9^{\mathrm{a}}$ & $18.5 \pm 0.8^{\mathrm{a}}$ & $2.8 \pm 0.9^{\mathrm{a}}$ & $21.3 \pm 1.7^{\mathrm{a}}$ & $2.4 \pm 0.2^{\mathrm{a}}$ \\
Biceps femoris & $5.4 \pm 1.3^{\mathrm{b}}$ & $16.9 \pm 1.5^{\mathrm{ab}}$ & $3.6 \pm 0.5^{\mathrm{a}}$ & $20.5 \pm 1.8^{\mathrm{a}}$ & $2.6 \pm 0.3^{\mathrm{a}}$ \\
\hline
\end{tabular}

a,b,c Superíndices diferentes dentro de columnas indican diferencias significativas $(p<0.05)$

\section{Conclusiones}

- Los músculos de apoyo (Longissimus thoracis y Psoas major) fueron más tiernos que los músculos locomotores (Semitendinosus, Quadriceps femoris, Biceps femoris).

- Los músculos con mayor capacidad de retención de agua fueron Semitendinosus y Infraspinatus.

\section{Literatura Cittada}

1. Almonacid MI. 2003. Estudio de $\mathrm{pH}$ y color muscular en cortes comerciales de canales bovinas normales y con anoma- lías de «Corte oscuro». Tesis de Pregrado. Valdivia, Chile: Escuela de Medicina Veterinaria, Universidad Austral de Chile. 53 p.

2. [AMSA] American Meat Science Association. 1995. Research guidelines for cookery and sensory evaluation and instrumental tenderness measurement of fresh meat. Chicago, USA: AMSANLSMB. American Meat Science Association in cooperation with National Livestock and Meat Board. 47 p.

3. Apaoblaza A, Pulido R, Gallo C. 2008. Efecto de una suplementación en base a maíz roleado, sobre la prevención de corte oscuro (CO) y algunas variables de calidad de la carne en novillos. En: XXXIII Congreso Anual de la Sociedad 
Chilena de Producción Animal. Valdivia, Chile. p 251-252.

4. Bass PD, Scanga JA, Chapman PL, Smith GC, Belk KE. 2009. Associations between portion size acceptability of beef cuts and ribeye area of beef carcasses. J Anim Sci 87: 2935-2942.

5. Belew JB, Brooks JC, McKenna DR, Savell JW. 2003. Warner-Bratzler shear evaluations of 40 bovine muscles. Meat Sci 64: 507-512.

6. Beltrán JA, Roncalés P. 2000. Determinación de la textura. En: Cañeque $\mathrm{V}$, Sañudo C (eds). Metodología para el estudio de la calidad de la canal y de la carne en rumiantes. Madrid: Instituto Nacional de Investigación y Tecnología Agraria y Alimentaria. p 168-174.

7. Cebra CK, Tornquist SJ, Van Saun RJ, Smith BB. 2001a. Glucose tolerance testing in llamas and alpacas. Am J Vet Res 62: 682-686.

8. Cebra CK, McKane SA, Tornquist SJ. 2001b. Effects of exogenous insulin on glucose tolerance in alpacas. Am J Vet Res 62: 1544-1547.

9. Cristofanelli S, Antonini A, Torres D, Polidori P, Renieri C. 2004. Meat and carcass quality from Peruvian llama (Lama glama) and alpaca (Lama pacos). Meat Sci 66: 589-593.

10. Duarte MS, Paulino PVR, Fonseca MA, Diniz LL, Cavali J, Serao NVL, Gomide LAM, et al. 2011. Influence of dental carcass maturity on carcass traits and meat quality of Nellore bulls. Meat Sci 88: 441-446.

11. Duckett SK, Neel JPS, Sonon RN, Fontenot Jr JP, Clapham WM, Scaglia G. 2007. Effects of winter stocker growth rate and finishing system on: II. Ninth tenth eleventh-rib composition, muscle color and palatability. J Anim Sci 85: 2691-2698.

12. Gallo C. 2010. La calidad de las canales y su carne. Informativo sobre carne y productos cárneos. N. ${ }^{\circ} 39$. Valdivia, Chile: Facultad de Ciencias Veterinarias, Universidad Austral de Chile. 74 p.
13. Herrera CA. 2008. Análisis descriptivo de factores asociados a la presentación de contusiones y $\mathrm{pH}$ elevado en canales de bovinos de distintas procedencias geográficas. Tesis de Pregrado. Valdivia, Chile: Escuela de Medicina Veterinaria, Universidad Austral de Chile. $48 \mathrm{p}$.

14. Hood D, Tarrant P. 1980. The problem of dark-cutting in beef. The Hague, Netherlands: Martinus Nijhoff. 504 p.

15. Jeremiah LE, Dugan MER, Aalhus JL, Gibson LL. 2003. Assessment of the chemical and cooking properties of the major beef muscles and muscle groups. Meat Sci 65: 985-992.

16. Lawrie RA, Ledward DA. 2006. Lawrie's meat science. $7^{\text {th }}$ ed. Cambridge, England: Woodhead Publishing. 521 p.

17. Lee JH, Kannan G, Eega KR, Kouakou B, Getz WR. 2008. Nutritional and quality characteristics of meat from goats and lambs finished under identical dietary regime. Small Rum Res 74: 255-259.

18. Muchenje V, Dzama K, Chimonyo M, Strydom P, Hugo A, Raats J. 2009. Some biochemical aspects pertaining to beef eating quality and consumer health: A review. Food Chem 112: 279-289.

19. Nakamura YN, Tsuneishi E, Kamiya M, Yamada A. 2010. Histological contribution of collagen architecture to beef toughness. J Food Sci 75: 73-77.

20. Orellana C, Peña F, García A, Perez, $J$, Martos J, Domenech V, Acero $R$. 2009. Carcass characteristics, fatty acid composition, and meat quality of Criollo Argentino and Braford steers raised on forage in a semi-tropical region of Argentina. Meat Sci 81: 57-64.

21. Pearce KL, Rosenvold K, Andersen HJ, Hopkins DL. 2011. Water distribution and mobility in meat during the conversion of muscle to meat and ageing and the impacts on fresh meat quality attributes. Areview. Meat Sci 89: 111-124. 
22. Polidori P, Renieri C, Antonini $M$, Passamonti P, Pucciarelli F. 2007. Meat fatty acid composition of llama (Lama glama) reared in the Andean highlands. Meat Sci 75: 356-358.

23. Priolo A, Micol D, Agabriel J, Prache $S$, Dransfield E. 2002. Effect of grass or concentrate feeding systems on lamb carcass and meat quality. Meat Sci 62: 179-185.

24. Realini CE, Duckett SK, Brito GW, Dalla Rizza M, De Mattos D. 2004. Effect of pasture vs. concentrate feeding with or without antioxidants on carcass characteristics, fatty acid composition, and quality of Uruguayan beef. Meat Sci 66: 567-577.

25. Salvá BK, Zumalacárregui JM, Figueira AC, Osorio MT, Mateo J. 2009. Nutrient composition and technological quality of meat from alpacas reared in Peru. Meat Sci 82: 450-455.
26. Savage AW, Warriss $P$ D, Jolley PD. 1990. The amount and composition of the proteins in drip from stored pig meat. Meat Sci 27: 289-303.

27. Tarrant PV, Grandin T. 1993. Cattle transport. In: Livestock handling and transport. UK: CAN International. p 109126.

28. [UNECE] United Nations Economic Commission for Europe. 2006. Llama/ alpaca meat carcases and cuts. New York: United Nations. 44 p.

29. Wirth F. 1987. Tecnología para la transformación de carne de calidad anormal. Fleischwirtschaft español 1: 22-28.

30. Wright LI, Scanga JA, Belk KE, Engle TE, Tatum JD, Person RC, et al. 2005. Benchmarking value in the pork supply chain: Characterization of US pork in the retail marketplace. Meat Sci 71:451-463. 